\title{
Klinische Studie zur Wirksamkeit und Verträglichkeit eines Medizinproduktes zur Prophylaxe des Auftretens akuter Symptome bei Patienten mit Hämorrhoidalleiden
}

\author{
Clinical Efficacy and Tolerability Study of a Medical Device for Prophylaxis \\ of the Occurrence of Acute Symptoms in Patients with Hemorrhoidal Disease
}

Autoren

Institute
R. Williams ${ }^{1}$, W. Havemeister ${ }^{1}$, M. Süßkind ${ }^{2}$, W. Wigger-Alberti ${ }^{1}$

${ }^{1}$ bioskin $\mathrm{GmbH}$, Hamburg/Berlin (Untersuchungszentren)

${ }^{2}$ DR. KADE Pharmazeutische Fabrik GmbH, Berlin

\section{Bibliografie}

Dol http://dx.doi.org/

10.1055/s-0033-1344505

Online-Publikation: 8.8.2013

Akt Dermatol 2013; 39: 504-508

(c) Georg Thieme Verlag KC

Stuttgart · New York

ISSN 0340-2541

Korrespondenzadresse

Dr. med.

Walter Wigger-Alberti

bioskin $\mathrm{GmbH}$

Burchardstraße 17

20095 Hamburg

walter.wigger@bioskin.de

\section{Zusammenfassung \\ $\nabla$}

Hintergrund: Topische Zubereitungen werden häufig in der Behandlung von symptomatischen Hämorrhoiden sowie begleitend zur Kausaltherapie eingesetzt.

Ziel: Ziel der vorliegenden Untersuchung war es, die Wirksamkeit und Verträglichkeit eines Medizinproduktes in der Langzeitbehandlung nachzuweisen.

Methoden: 100 Patienten wurden in zwei Gruppen randomisiert: Gruppe A behandelte sich in zwei Intervallen von jeweils vier Wochen Dauer mit einem Medizinprodukt, Gruppe B blieb unbehandelt. Beide Gruppen dokumentierten täglich das Auftreten und die Stärke der am meisten beeinträchtigenden Symptome (most bothersome symptom $=\mathrm{MBS}$ ) sowie die Applikation der ausgegebenen Akutmedikation. Gruppe A beantwortete einen Fragebogen zur Produktwirksamkeit, Verträglichkeit und anderen Eigenschaften. An den Tagen 1, 29 und 57 erfolgten klinische und subjektive Bewertungen der Symptome.

\section{Einleitung}

Unter Hämorrhoiden versteht man die Vergrößerung und nachfolgende Absenkung des Plexus haemorrhoidalis superior, der physiologisch oberhalb der Linea dentata und bedeckt von der Rektummukosa gelegen ist [1,2]. Verursachen die Hyperplasie und eine mögliche Entzündung der darüber liegenden Schleimhaut Beschwerden, so wird von einem Hämorrhoidalleiden gesprochen [3-5]. Die Symptome können die Lebensqualität sehr stark beeinträchtigen. So vermuten Historiker, dass Napoleon Bonaparte die Schlacht bei Waterloo verloren hat, weil er schon seit 1814 und insbesondere am 18.Juni 1815 unter so schmerzhaften Hämorrhoiden litt, dass sein Geschick als Feldherr infolge des Schlafentzugs und
Ergebnisse: Das Auftreten und die Intensität von Juckreiz und Brennen nahmen in der Gruppe A über den Behandlungszeitraum kontinuierlich ab, in der unbehandelten Gruppe zeigte sich nur eine sehr leichte Abnahme. Die Scoresumme der täglichen MBS-Bewertung (Tag 1-57) war in der Gruppe A im Vergleich zur Gruppe B statistisch signifikant geringer. Auch in der Frequenz und der mittleren Dauer der akuten subjektiven Symptom-Episoden sowie der Häufigkeit der Anwendung der Akutmedikation zeigten sich statistisch signifikante Unterschiede. In Gruppe A wurde eine leichte Abnahme von Ödem und Erythem festgestellt, in Gruppe B nur geringfügige Änderungen. Die meisten Patienten gaben an, dass die Salbe ihnen geholfen hat, bewerteten die Gesamtwirksamkeit und Verträglichkeit als „sehr gut" bis „gut“ und würden das Produkt regelmäßig anwenden und weiterempfehlen.

Schlussfolgerung: Eine Hämorrhoidalsalbe mit Hautschutzkomplex lindert nicht nur typische akute Beschwerden, sondern verlängert auch die beschwerdefreien Intervalle und verringert somit signifikant den Gebrauch einer Akutmedikation.

der Einnahme von Opiumtropfen zur Schmerzlinderung negativ beeinflusst war [6,7].

Hämorrhoidalleiden sind weit verbreitet, weshalb auch von einer "Volkskrankheit“ gesprochen wird [4,8-11]. Schätzungsweise $50 \%$ der Erwachsenen sind im Laufe ihres Lebens mindestens einmal betroffen $[12,13]$, wenngleich valide epidemiologische Studien zur Prävalenz von Hämorrhoiden fehlen [14]. Die Angaben aus zugänglichen Studien streuen diesbezüglich erheblich. Kürzlich wurden Daten einer prospektiven Studie von 976 österreichischen Patienten, die sich im Rahmen der Krebsvorsorge einer Koloskopie unterzogen, publiziert [15]. Bei insgesamt 380 Patienten $(38,9 \%)$ wurden vergrößerte Hämorrhoiden diagnostiziert, von denen sich die Mehrzahl (72,9\%) im Stadium I, 18,4\% im Stadium II, 
8,2 \% im Stadium III und nur 0,5\% der Patienten im Stadium IV befanden. 44,7\% dieser Patienten klagten über Symptome, die im Zusammenhang mit den diagnostizierten Hämorrhoiden standen. Die auch in dieser Studie zugrunde gelegte Einteilung der Stadien in vier Grade erfolgt mehrheitlich nach Goligher [16] entsprechend dem jeweiligen Erscheinungsbild von Lokalisation und Ausmaß des Prolaps. Dabei sind innere Hämorrhoiden, die dem Grad I entsprechen, nur durch die proktoskopische Untersuchung und nicht rektal-digital zu diagnostizieren. Hämorrhoiden II. Grades prolabieren beim Stuhlgang und ziehen sich anschließend wieder zurück. Hämorrhoiden III. Grades ziehen sich nach der Defäkation nicht mehr spontan zurück, sondern müssen manuell reponiert werden. Bei Grad IV lassen sich die Hämorrhoiden nicht mehr reponieren, der Prolaps ist fixiert $[2,17]$. Darüber hinaus existieren allerdings eine Vielzahl unterschiedlicher Definitionen der vier Grade $[3,18]$.

Die Ätiologie, Pathogenese und spezifische Symptomatik von Hämorrhoiden werden noch immer kontrovers diskutiert [2, 3,14,19-21]. Mehrheitlich werden eine ballaststoffarme Ernährung, falsches Stuhlverhalten (sogenanntes „Nachpressen“), der Missbrauch von Laxantien sowie eine genetische Disposition zur Bindegewebeschwäche als Hauptauslöser von Hämorrhoiden genannt [12]. In einer Metaanalyse von sieben randomisierten kontrollierten Studien konnte die positive Wirkung ballaststoffreicher Ernährung belegt werden [22].

Das klassische Symptom eines Hämorrhoidalleidens insbesondere im Frühstadium sind hellrote Blutauflagerungen auf dem Stuhl. Oft nimmt der Betroffene dies nur als Blut auf dem Toilettenpapier wahr. Viele Patienten leiden aber auch unter analem Juckreiz und Brennen. Die Hämorrhoiden selbst jucken nicht, vielmehr gilt Juckreiz als Zeichen dafür, dass die hochsensible Analschleimhaut gereizt und teilweise entzündet ist. Ursächlich dafür kann eine gestörte Feinkontinenz im Rahmen des Hämorrhoidalleidens sein [23]. Die häufigsten Beschwerden sind Blutungen (ca. $70 \%$ ) und Juckreiz (60-70\%), gefolgt von Nässen (ca. 40\%) und Brennen (ca. 40\%) [1,24,25]. Trotz typischer Symptome ist es unbedingt erforderlich, andere Ursachen der Beschwerden auszuschließen und eine zuverlässige Diagnostik vorzunehmen [3]. Ohne Therapie ist beim Hämorrhoidalleiden meist mit einer Progredienz der Beschwerden und der Ausprägung zu rechnen. Lokaltherapeutika, auch Proktologika genannt, wirken zwar nicht kausal, können aber die bei Hämorrhoiden auftretenden entzündlichen bzw. ödematösen Begleiterscheinungen günstig beeinflussen [2]. Langfristig nachweisbare und wirksame Therapien stellen die Sklerosierungsbehandlung, die Gummibandligatur, die thermische Koagulation sowie segmentäre und zirkuläre Operationsverfahren dar [2]. Unerlässlich ist zudem eine Basistherapie bestehend aus einer Ernährungsberatung zur Stuhlregulation, das Vermeiden übermäßigen Pressens während der Defäkation, tägliche körperliche Betätigungen im Sinne von Spaziergängen und Sport sowie angemessene Hygienemaßnahmen (z.B. Reinigung mit klarem Wasser) [1,25]. Je nach Ausprägungsgrad stehen somit die reine Symptombehandlung, die Kausaltherapie oder eine Rezidivprophylaxe im Vordergrund.

Topische Zubereitungen werden vielfach in der Behandlung von rein symptomatischen Hämorrhoiden sowie begleitend zur Kausaltherapie eingesetzt, wenngleich gut kontrollierte Studien selten sind und ihr therapeutischer Wert intensiv diskutiert wird [12,26,27]. Es werden Cremes, Salben und sogenannte Analtampons mit z.T. anästhesierenden, antiphlogistischen und/oder adstringierenden Zusätzen verordnet. Auch Glukokortikoide und Antimykotika kommen zum Einsatz [12,28].
Drei Symptomenkomplexe sind einer topischen Therapie zugänglich: 1.Irritationen der Perianalhaut wie Jucken, Brennen, Rötung und Wundsein, 2.Beschwerden im distalen Analkanal mit Schmerzen beispielsweise im Rahmen einer Anitis, Kryptitis oder Papillitis sowie 3.Thrombosen der perianalen, intermediären und hämorrhoidalen Geflechte [24].

In einer kleinen Pilotstudie wurden positive Auswirkungen einer Bienenwachs, Olivenöl und Honig enthaltenden Formulierung auf Blutungen und Juckreiz von Patienten mit Hämorrhoiden berichtet [29]. Kürzlich wurden Ergebnisse einer plazebo-kontrollierten doppelblinden Studie publiziert, die für ein topisches Medizinprodukt, welches Hyaluronsäure, Methylsulfonylmethan und Ölextrakt vom Australischen Teebaum (Melaleuca alternifolia) enthält, eine signifikante Reduktion subjektiver Beschwerden wie Schmerz und Pruritus gegenüber einer Behandlung mit Plazebo zeigte [30]. Darüber hinaus gibt es Daten zum Nutzen von topischem Glyceryltrinitrat bei Patienten mit geringgradigen Hämorrhoiden zur Senkung des Drucks im Analkanal [31], von topischem Nifedipin bei akut thrombosierten Hämorrhoiden [32] sowie von topischem Phenylephrin zur Vasokonstriktion [33].

Ziel der vorliegenden Untersuchung war, die Wirksamkeit und Verträglichkeit eines Medizinproduktes (Posterisan ${ }^{\circledR}$ protect, DR. KADE, Berlin) in der Langzeitbehandlung zu untersuchen. Das Präparat enthält einen Hautschutzkomplex bestehend aus Jojobawachs, gelbem Bienenwachs und Cetylstearylisononanoat. Jojobawachs pflegt und schützt durch die Bildung eines dünnen Lipidfilms. Bienenwachs ist wasserabweisend und Cetylstearylisononanoat erhöht die Geschmeidigkeit der Haut. Durch den Schutzmantel soll die empfindliche Analschleimhaut vor weiteren Reizungen geschützt werden. Gleichzeitig wird der Stuhlgang durch den mechanischen Gleiteffekt erleichtert. Insgesamt soll das Präparat nicht nur Beschwerden lindern, sondern auch vor dem Wiederauftreten von akuten Hämorrhoidalsymptomen schützen. Daher sollte untersucht werden, ob die Salbe in der Lage ist, das Auftreten und die Stärke von subjektiven Symptomen bei Patienten mit Hämorrhoidalleiden zu verringern und deren Beschwerde-Episoden zu verkürzen.

\section{Methoden \\ $\nabla$}

\section{Patienten}

Die klinische Untersuchung wurde randomisiert, im Parallelgruppendesign bei der bioskin $\mathrm{GmbH}$, zweizentrisch in Hamburg und Berlin, von Februar bis Juni 2012 durchgeführt. Die Rekrutierung der Patienten erfolgte gemäß den Anforderungen nach ISO 14155 und der Helsinki-Deklaration. Die Studiendokumente wurden von der federführenden Ethikkommission (Ärztekammer Hamburg) genehmigt sowie von der involvierten Ethikkommission Berlin anerkannt.

Hundert männliche und weibliche Patienten (50 je Gruppe) im Alter von 18 Jahren oder älter mit Hämorrhoiden des Schweregrades I oder II in der Anamnese und mindestens drei akuten Symptom-Episoden innerhalb der acht Wochen vor Untersuchungseinschluss gaben ihre schriftliche Einwilligung und wurden randomisiert (Gruppe A, Anwendung von Posterisan ${ }^{\circledR}$ protect: 24 Männer, 26 Frauen, mittleres Alter=53,1 Jahre; Gruppe B, unbehandelt: 26 Männer, 24 Frauen, mittleres Alter=48,8 Jahre). Hauptausschlusskriterien waren: Hämorrhoiden mit Schweregrad III oder IV, ähnliche Symptome in der Analregion, welche nicht durch Hämorrhoiden verursacht wurden (z.B. Marisken 
oder Dermatosen), Schwangerschaft und Stillzeit, bestehende Unverträglichkeitsreaktionen auf Inhaltsstoffe des Prüfpräparates sowie symptomatische oder kausale Therapien vor Einschluss in die Studie.

\section{Studiendesign}

Nach der Randomisierung behandelten sich die Patienten der Gruppe A in zwei Intervallen von jeweils vier Wochen Dauer mit Posterisan ${ }^{\circledR}$ protect. Die Applikation erfolgte mit dem Finger bzw. im Analkanal mittels eines Applikators. Die Patienten der Gruppe B blieben unbehandelt. Beide Gruppen dokumentierten täglich mittels einer Skala von 0 bis 10 das Auftreten und die Stärke der am meisten beeinträchtigenden Symptome (most bothersome symptom =MBS) in einem Tagebuch. Weiterhin mussten beide Gruppen die Applikation der ebenfalls ausgegebenen Lidocainhaltigen Akutmedikation (Posterisan ${ }^{\circledR}$ akut Rektalsalbe, DR. KADE, Berlin) dokumentieren, welche im Fall eines MBS-Scores $\geq 8$ angewendet werden durfte. Des Weiteren mussten die Patienten der Gruppe A einen Fragebogen zur Produktwirksamkeit, Verträglichkeit und anderen Eigenschaften beantworten. An den Tagen 1, 29 und 57 erfolgte eine klinische Bewertung durch den Prüfarzt mittels einer 5-Punkte-Skala (0-4) und einer subjektiven Bewertung der Symptome durch die Patienten.

\section{Klinische Bewertung}

Für die tägliche Bewertung des MBS wurde eine numerische Skala von 0 bis 10 (nicht vorhanden bis sehr stark) verwendet. Als MBS wurde eines der Symptome (Juckreiz, Brennen, Schmerzen unabhängig vom Stuhlgang oder Schmerzen während des Stuhlgangs), welches das individuell am meisten beeinträchtigende Symptom beim jeweiligen Patienten war, definiert. Die numerische Skala war in vier Kategorien klassifiziert: 0 (Symptome nicht vorhanden); 1-4 (leichte Symptome); 5-7 (mäßige Symptome); 8-10 (schwere Symptome).

Die klinische Bewertung der Analregion durch den Arzt erfolgte an den Tagen 1, 29 und 57 mittels 5-Punkte-Skala (0-4) anhand der folgenden Parameter: Ödem, Erythem, Blutungen und Fissuren.

\section{Statistik}

Der Vergleich der Scoresummen des MBS beider Gruppen über den Zeitraum Tag 1-57 erfolgte mittels Student's t-Tests sowie einer deskriptiven Analyse des mittleren Behandlungseffekts.

Zudem wurden beide Gruppen hinsichtlich der Frequenz der akuten subjektiven Symptom-Episoden, der mittleren Dauer der akuten subjektiven Symptom-Episoden sowie der Häufigkeit der Anwendung der Akutmedikation Posterisan ${ }^{\circledR}$ akut mittels Mann-Whitney-U-Tests verglichen. Weiterhin erfolgte eine Schätzung des Behandlungseffekts mittels Hodges-Lehman und dessen 95\%-Konfidenzintervalls. Alle anderen Parameter wurden deskriptiv ausgewertet.

\section{Ergebnisse}

$\nabla$

Die Daten aller 100 Patienten waren valide für das Safety-evaluation-set (SES) und das Full-analysis-set (FAS). Das SES enthielt alle randomisierten Probanden und alle Analysen zur Sicherheitsbewertung basierten auf dieser Population. Das FAS enthielt alle randomisierten Probanden mit zumindest einer Post-baseline-Bewertung, und alle „Intent-to-treat“-Analysen basierten auf dieser Population. 47 Patienten der Gruppe A und alle $50 \mathrm{~Pa}-$ tienten der unbehandelten Gruppe B wurden in das Valid-casesset (VCS) eingeschlossen. Drei Patienten wurden aufgrund von Protokollverletzungen aus der VCS ausgeschlossen. Das VCS enthielt alle Probanden ohne wesentliche Abweichungen vom klinischen Untersuchungsplan (einschließlich Einschlusskriterien) und mit verfügbaren Werten der primären Variablen an allen Tagen, ohne kalkulierte Werte. Die „Per-protocol“-Analysen basierten auf dieser Population.

Nahezu alle eingeschlossenen Patienten berichteten, dass sie seit mehreren Jahren unter Hämorrhoidalbeschwerden litten. Etwa drei Viertel (74\%) suchten Rat bei Freunden, gefolgt von $42 \%$ der Patienten, die sich über die Apotheke informierten. Einige bezogen Rat über das Internet (18\%), einige suchten Ärzte auf (14\%) und $8 \%$ informierten sich anderweitig. Die meisten Patienten $(>60 \%)$ empfanden ihr Problem am stärksten ausgeprägt über den Tag und während des Stuhlgangs. 24\% klagten über Beschwerden während der Nacht. Jeweils 10\% empfanden ihr Problem am stärksten ausgeprägt während des Sports, der Arbeit oder in ihrer Partnerschaft. Die Mehrheit der Patienten erwartete, dass das Produkt Beschwerden lindert (84\%) und einem erneuten Auftreten der Beschwerden vorbeugt (74\%). 44\% erwarteten, dass das Produkt vor Reizungen schützt und $24 \%$, dass die Analregion gepflegt wird. 83,3\% hatten bereits andere Produkte in der Vergangenheit verwendet.

Zu Untersuchungsbeginn (Baseline) litten die meisten Patienten beider Gruppen unter überwiegend leichtem bis mäßigem Juckreiz (Gruppe A: 80\%, Gruppe B: 78\%) und Brennen (Gruppe A: 76\%, Gruppe B: 70\%). Der mittlere Score für Juckreiz betrug 3,5 bzw. 3,9. Der mittlere Score für Brennen betrug 3,3 bzw. 2,6. Über den Behandlungszeitraum nahm das Auftreten und die Intensität von Juckreiz und Brennen in der Gruppe A kontinuierlich ab. Am Ende der Untersuchung (Tag 57) betrug der mittlere Score für Juckreiz 1,5 und der mittlere Score für Brennen 0,8 . In der unbehandelten Gruppe wurde nur eine sehr leichte Abnahme von Juckreiz und Brennen über den Untersuchungszeitraum dokumentiert. Der mittlere Score für Juckreiz am Tag 57 betrug 3,3 und der mittlere Score für Brennen 2,1.

\section{MBS}

$\nabla$

Die Scoresumme der täglichen MBS-Bewertung über den Zeitraum Tag 1-57 war statistisch signifikant geringer $(p \leq 0,0001)$ in der Gruppe A im Vergleich zur unbehandelten Gruppe B (FAS: 171,46 vs. 249,62 ; VCS: 170,17 vs. 249,62 ) ( Abb. 1 ).

Weitere Analysen zeigten statistisch signifikante Unterschiede zwischen der Gruppe A und der unbehandelten Gruppe B hinsichtlich der Frequenz der akuten subjektiven Symptom-Episoden (FAS: Mittelwert: 2,04 vs. 3,60; p=0,0004) ( $\bullet$ Abb.2), der mittleren Dauer der akuten subjektiven Symptom-Episoden (FAS: Mittelwert: 5,29 vs. 9,26; $p=0,0027$ ) sowie der Häufigkeit der Anwendung der Akutmedikation (FAS: Mittelwert: 0,52 vs. $1,70 ; p=0,0010)$.

\section{Klinische Bewertung durch den Arzt}

Der mittlere Score für Ödem betrug 0,9 für beide Behandlungsgruppen. Der mittlere Score für Erythem betrug 1,4 für Gruppe A und 1,1 für die unbehandelte Gruppe. Fissuren wurden bei vier Patienten (8\%) der Gruppe A, aber bei keinem Patienten der unbehandelten Gruppe zu Untersuchungsbeginn beschrieben. Am Ende der klinischen Untersuchung (Tag 57) der Gruppe A betrug der mittlere Score für Ödem 0,4 und der mittlere Score für 


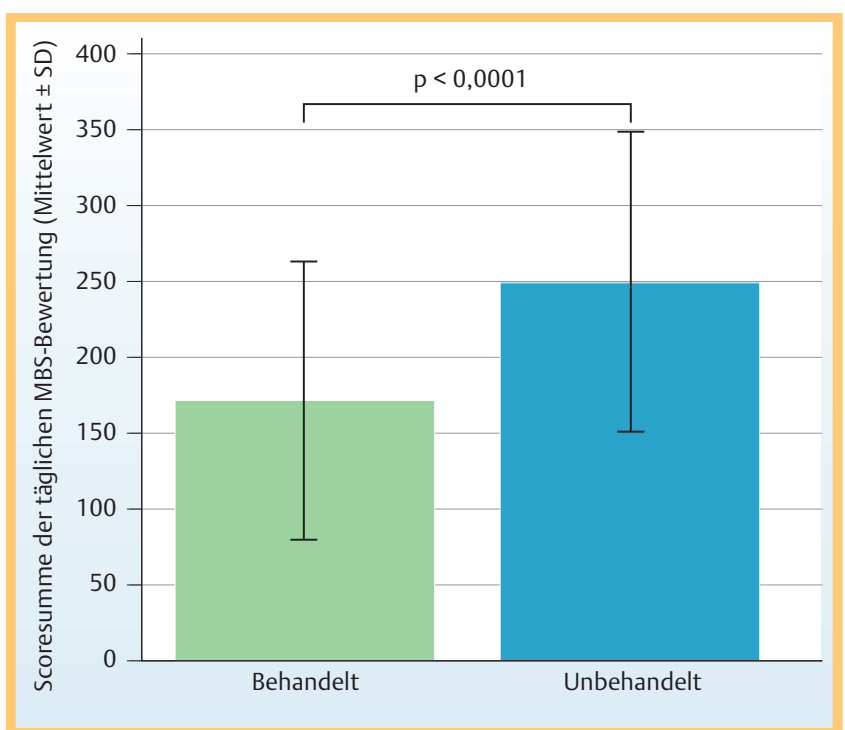

Abb. 1 Mittlere Scoresumme $( \pm S D)$ der täglichen MBS-Bewertung (mittels Patiententagebuch) über den Behandlungszeitraum Tag 1-57. Der statistische Vergleich behandelt vs. unbehandelt zeigte eine signifikant geringere mittlere Scoresumme nach Behandlung mit Posterisan ${ }^{\circledR}$ protect (171,46 vs. 249,62; $p<0,0001)$. Die Bewertung des MBS erfolgte mittels numerischer Skala von 0 bis 10 (nicht vorhanden bis sehr stark). Als MBS wurde eines der Symptome (Juckreiz, Brennen, Schmerzen unabhängig vom Stuhlgang oder Schmerzen während des Stuhlgangs), welches das individuell am häufigsten und beeinträchtigendste Hämorrhoiden-Symptom beim jeweiligen Patienten war, definiert.

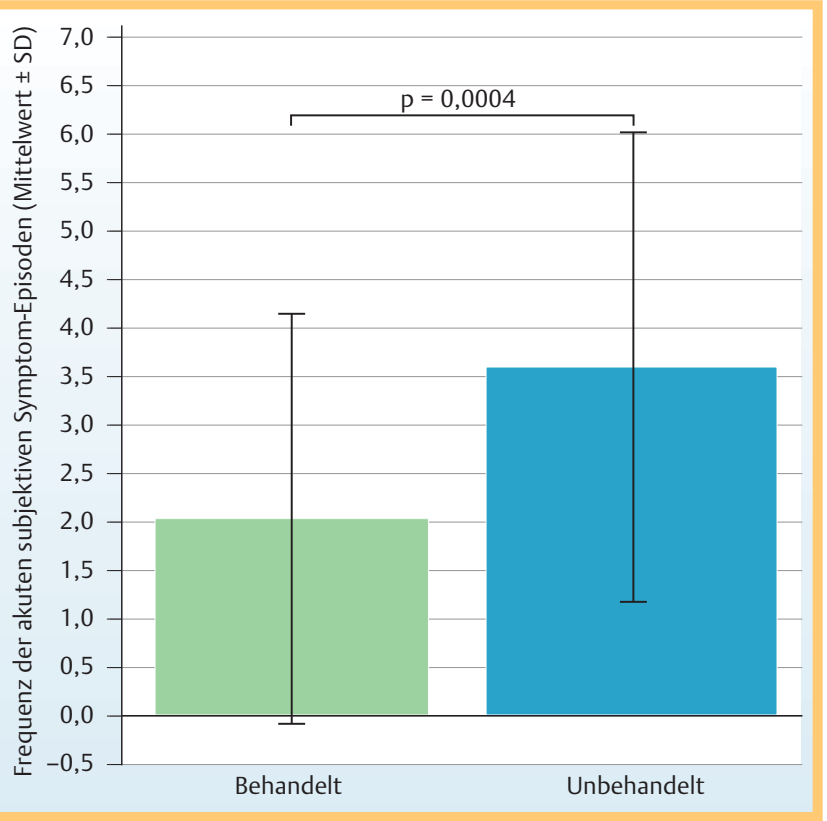

Abb.2 Mittlere Frequenz der akuten subjektiven Symptom-Episoden $( \pm$ SD), definiert als kontinuierliches Auftreten ( $\geq 1 \mathrm{Tag}$ ) von MBS mit zumindest mäßigem Schweregrad über den Behandlungszeitraum Tag 1-57. Der statistische Vergleich behandelt vs. unbehandelt zeigte eine signifikant geringere mittlere Frequenz nach Behandlung mit Posterisan ${ }^{\circledR}$ protect $(2,04$ vs. 3,$60 ; p=0,0004)$.
Erythem 0,6. Leichte Fissuren wurden für einen Patienten der Gruppe A am Ende der klinischen Untersuchung beschrieben. Nur geringfügige Änderungen zeigten sich für Ödem und Erythem über den gesamten Untersuchungszeitraum in der unbehandelten Gruppe B.

\section{Fragebogen}

Am Ende des Behandlungszeitraums bestätigten 60,4\% der Patienten der Gruppe A die Aussage das Produkt „lindert Beschwerden“. Die Aussagen „schützt vor Reizungen“, „pflegt die Analregion“ und „beugt dem erneuten Auftreten der Beschwerden vor“ wurden von etwa 20-40\% der Patienten bestätigt. Am Ende der Untersuchung berichteten die meisten Patienten (87,5\%), dass die Salbe ihnen geholfen hat, und stuften die Gesamtwirksamkeit $(83,3 \%)$ und die Verträglichkeit (95,9\%) am Anwendungsort als „sehr gut“ bis „gut“ ein. Insgesamt würden nahezu alle Patienten das Produkt regelmäßig anwenden (89,6\%) und weiterempfehlen $(91,7 \%)$.

\section{Diskussion}

Eine topische Therapie des Hämorrhoidalleidens durch die Applikation von Salben und Suppositorien ist nicht kurativ, kann aber die subjektiv sehr starken Beschwerden der Patienten lindern. Die Daten unserer Studie zeigen, wie stark betroffene Patienten durch die typischen Symptome beeinträchtigt sind. Über $60 \%$ der befragten Patienten empfanden ihr Problem am meisten beeinträchtigend über den Tag und während des Stuhlgangs. Damit verbunden hat die Mehrheit der betroffenen Patienten die Erwartung, dass ein Produkt zur Behandlung von Hämorrhoiden die subjektiven Beschwerden lindert und einem erneuten Auftreten der Beschwerden vorbeugt. Daher war das Hauptaugenmerk einerseits auf die subjektiven Beschwerden und anderseits auf die Frequenz und Dauer der akuten subjektiven Symptome und somit die Häufigkeit der Anwendung der zur Verfügung gestellten Akutmedikation gerichtet.

Die subjektive und die klinische Bewertung zeigten über den Untersuchungszeitraum eine kontinuierliche Abnahme von Juckreiz und Brennen sowie von Erythem und Ödem. Bei den meisten Patienten, die zu Untersuchungsbeginn über Schmerzen unabhängig oder während des Stuhlgangs berichteten, waren nach Behandlung mit dem Prüfpräparat die Schmerzen abgeschwächt oder nicht mehr vorhanden. In der unbehandelten Gruppe waren im Gegensatz nur ganz geringfügige Änderungen dieser Parameter zu sehen. Der Unterschied zwischen der behandelten und unbehandelten Gruppe war statistisch signifikant. Die Ergebnisse decken sich mit den eingangs genannten Erwartungen der Patienten, dass durch Anwendung eines topischen Proktologikums die Frequenz und Dauer der akuten Symptom-Episoden signifikant gesenkt werden und seltener zu einer Akutmedikation gegriffen werden muss.

Das Konzept, mittels eines Hautschutzkomplexes die empfindliche Analschleimhaut zu schützen und durch eine erhöhte Gleitfähigkeit eine schmerzarme Defäkation zu erreichen, scheint vielversprechend zu sein. Da das Beschwerdemaximum beim Stuhlgang besteht, ist der subjektive Nutzen dieser Therapieoption für die Patienten so groß, dass am Ende der Untersuchung eine hohe Zustimmungsrate zur Wirksamkeit erzielt wurde. Der Nutzen der physikalischen Eigenschaften eines topischen Medizinproduktes zum Schutz der Analhaut und zur Erleichterung der Defäkation wurde kürzlich in einer Studie mit kleiner Fallzahl 
belegt [30]. Erstmalig konnte mit der hier vorliegenden Studie gezeigt werden, dass die Anwendung eines Medizinproduktes bei Hämorrhoidalleiden klar zu einer Senkung der Rezidivhäufigkeit führte.

Zusammenfassend wurde in der Studie nachgewiesen, dass die topische Therapie mit der einen Hautschutzkomplex enthaltenden Hämorrhoidalsalbe nicht nur typische akute Beschwerden lindert, sondern auch beschwerdefreie Intervalle verlängert und signifikant den Gebrauch einer Akutmedikation verringern hilft.

\section{Interessenkonflikt}

R. Williams, W. Havemeister und W. Wigger-Alberti sind Mitarbeiter der bioskin $\mathrm{GmbH}$, Hamburg/Berlin, M. Süßkind ist Mitarbeiterin der DR. KADE Pharmazeutische Fabrik GmbH, Berlin.

\section{Abstract}

\section{Clinical Efficacy and Tolerability Study of a Medical Device for Prophylaxis of the Occurrence of Acute Symptoms in Patients with Hemorrhoidal Disease}

Background: Topical preparations are intended for use in symptomatic hemorrhoids or are applied as concurrent causal therapy. Objective: The aim of this study was to prove the efficacy and tolerability of a medical device during long-term use.

Methods: 100 patients were randomized in two groups: Group A performed treatment with a medical device in two intervals of four weeks each, group B remained untreated. Both groups documented the occurrence and strength of the most bothersome symptom (MBS) once daily and the application of the dispensed acute medication. Group A completed a questionnaire on product efficacy, tolerability and other properties. On Days 1, 29 and 57 clinical and subjective assessments were performed.

Results: In group A, the occurrence and intensity of pruritus and burning has continually decreased over the treatment period whereas only a very slight decrease was seen in group $B$. The score sum of the daily MBS evaluation (Day 1 - 57) was statistically significant lower in group A when compared to group B. Statistically significant differences were also seen in frequency and mean duration of acute subjective symptom episodes as well as in frequency of use of acute medication. In group A, a slight decrease in edema and erythema was noted. In group B only minor changes were seen. Most of the patients reported that the ointment helped them, assessed the overall efficacy and tolerability as "very good" to "good" and would regularly apply and recommend the product.

Conclusion: A hemorrhoidal ointment with skincare complex soothes not only typical acute symptoms, it even prolongs symptom-free intervals and therefore significantly reduces the use of acute medication.

\section{Literatur}

1 Lenhard BH. Hämorrhoiden. Differenzialdiagnose und Therapie. 55. Heidelberg: Springer Verlag; 2004: 240-247

2 Joos AK, Herold A. Hämorrhoidalleiden. Neue konservative und operative Therapien für ein weit verbreitetes Leiden. Der Gastroenterologe. 5. Heidelberg: Springer Verlag; 2010: 326-335

3 Rohde H. Was sind Hämorrhoiden? Sammelbegriff, Symptom oder Erkrankung? Dtsches Ärztebl 2005; 102: A209-A213

4 Brisinda G. How to treat haemorrhoids. BMJ 2000; 321: $582-583$

5 Pfenninger JL. Modern treatments for internal haemorrhoids. BMJ 1997; 314: $1211-1212$

6 Welling DR, Wolff BG, Dozois RR. Piles of defeat. Napoleon at Waterloo. Diseases of the Colon \& Rectum 1988; 31: 303-305

7 Cronin V. Napoleon. Eine Biographie. Berlin: Claassen; 1973

8 Fazio VW, Tjandra JJ. The management of perianal diseases. Advances in Surg 1996; 29: 59-78

9 Giebel GD. Etiology, clinical aspects and therapy of hemorrhoid disease. Krankenpfl J April 1990; 28: 154-160

10 Metcalf A. Anorectal disorders. Five common causes of pain, itching, and bleeding. Postgrad Med 1995; 98: 0581 -94

11 Nagle $D$, Randelli RH. Primary care office management of perianal and anal disease. Primary Care 1996; 23: 609-620

12 Kirsch JJ. Medikamentöse Hämorrhoidenbehandlung. Therapiewoche 1989; 39: 800-804

13 Wienert V, Mlitz H. Atlas Koloproktologie. Berlin: Blackwell Wissenschafts-Verlag; 1997

14 Herold A. Studienadaptierte Therapie des Hämorrhoidalleidens. Chirurg 2008; 79: 418-429

15 Riss S, Weiser FAQ Schwameis Ket al. The prevalence of hemorrhoids in adults. Int J Colorectal Dis 2012; 27: 215 - 220

16 Goligher JC. Surgery of the anus, rectum and colon. 3rd ed. London: Baillière Tindall; 1975

17 Kirsch JJ, Grimm BD. Die konservative Hämorrhoidenbehandlung. Wien med Wochenschr 2004; 154: 50-55

18 Lunniss PJ, Mann CV. Classification of internal haemorrhoids: a discussion paper. Colorectal Dis 2004; 6: 226-232

19 Hyams L, Philpot J. An epidemiological investigation of hemorrhoids. Am J Proctol 1970; 21: 177-193

20 Mazier WP. Hemorrhoids, fissures, and pruritus ani. Surg Clin N Am 1994; 74: 1277 - 1299

21 Lohsiriwat V. Hemorrhoids: From basic pathophysiology to clinical management. World J Gastroenterol 2012; 18: 2009-2017

22 Alonso-Coello P, Mills E, Heels-Ansdell D et al. Fiber for the treatment of hemorrhoids complication: A systemic review and metaanalysis. Am J Gastroenterol 2006; 101: 181-188

$23 \mathrm{Krammer} \mathrm{HJ.} \mathrm{Hämorrhoidalleiden.} \mathrm{Ein} \mathrm{Update} \mathrm{für} \mathrm{die} \mathrm{Apothekenpraxis.}$ Pharmazeutische Zeitung 2010: 21

24 Kirsch JJ. Medikamentöse Behandlung des Hämorrhoidalleidens - Sinn und Unsinn in der Therapie. Therapiewoche 1996; 27: 1506-1513

25 Raulf F. Differentialdiagnostik und Therapie des Hämorrhoidalleidens. Kontinenz aktuell 2010: 5-10

26 Kirsch JJ, Wienert V. Positivliste „Hämorrhoidenmittel“. Coloproctology 2001; 23: $295-297$

27 Rohde $H$. Hämorrhoidenmittel. Placebos oder mehr? Dtsch Ärztebl 2002; 99: A1133

28 Johanson JF. Nonsurgical treatment of hemorrhoids. J Gastrointest Surg 2002; 6: 290-294

29 Al-Waili NS, Saloom KS, Al Waili TN et al. The safety and efficacy of a mixture of honey, olive oil, and beeswax for the management of hemorrhoids and anal fissure: A pilot study. The Scientific World Journal 2006; 6: 1998 -2005

30 Joksimovic N, Spasovski G, Joksimovic V et al. Efficacy and tolerability of hyaluronic acid, tea tree oil and methyl-sulfonyl-methane in a new gel medical device for treatment of haemorrhoids in double-blind, placebo-controlled clinical trial. Updates Surg 2012; 64: 195-201

31 Tjandra JJ, Tan JJ, Lim JF et al. Rectogesic (glyceryl trinitrate $0.2 \%$ ) ointment relieves symptoms of haemorrhoids associated with high resting anal canal pressures. Colorectal Dis 2007; 9: 457-463

32 Perrotti P, Antropoli C, Molino D et al. Conservative treatment of acute thrombosed external hemorrhoids with topical nifedipine. Dis Colon Rectum 2001; 44: 405-409

33 Sneider EB, Maykel JA. Diagnosis and management of symptomatic hemorrhoids. Surg Clin North Am 2010; 90: 17-32 